\title{
Linx
}

Revue des linguistes de l'université Paris X Nanterre

$7 \mid 1995$

Saussure aujourd'hui

\section{Le signe dans les discours technoscientifiques : deux hypothèses}

Yves Gentilhomme

\section{(2) OpenEdition}

1 Journals

Édition électronique

URL : http://journals.openedition.org/linx/1215

DOI : 10.4000/linx.1215

ISSN : 2118-9692

Éditeur

Presses universitaires de Paris Nanterre

\section{Édition imprimée}

Date de publication : 1 septembre 1995

Pagination : 353-368

ISSN : 0246-8743

\section{Référence électronique}

Yves Gentilhomme, "Le signe dans les discours technoscientifiques : deux hypothèses », Linx [En ligne], 7 | 1995, mis en ligne le 25 juillet 2012, consulté le 10 décembre 2020. URL : http:// journals.openedition.org/linx/1215 ; DOI : https://doi.org/10.4000/linx.1215

Ce document a été généré automatiquement le 10 décembre 2020.

Département de Sciences du langage, Université Paris Ouest 


\title{
Le signe dans les discours technoscientifiques : deux hypothèses
}

\author{
Yves Gentilhomme
}

1 Symboles et abréviations

\begin{tabular}{|l|l|}
\hline S = signe (triade) & Sling = système linguistique \\
\hline Sa = signifiant & Ssymb = système symbolique \\
\hline Sé = signifié & Ssém = système sémiologique mixte \\
\hline Snot = signifié notionnel (notion) & \\
\hline Sconc = signifié conceptuel (concept) & St = syntactique \\
\hline
\end{tabular}

\section{Avant-propos}

L'immense mérite de Saussure est, selon nous, d'avoir dégagé un certain nombre de concepts sémiologiques de base pour une prime approche heuristique, parmi les multiples fluctuations concomitantes, structurations d'une importance théorique certaine, mais que, méthodologiquement, il paraît plus judicieux de traiter comme des développements de second ordre.

2 Parmi ces concepts, nous portons notre attention sur celui de signe (mot ou symbole) et, conséquemment, sur les concepts qui lui sont organiquement associés (signifiant, signifié, arbitrarité, motivation, stabilité, etc.) fonctionnant dans un système sémiologique mixte : 
$3 \quad($ Ssém $)=$ système langue naturelle (Sling) $\cup$ système symbolique (Ssymb), nous fondant sur des discours technoscientifiques auxquels nous avons été confronté au cours de notre carrière, avec l'espoir que nos remarques puissent être étendues, moyennant des ajustements appropriés, à une base plus large de données textuelles [Gentilhomme.92] .

$4 \mathrm{Si}$, dans la production d'un discours tout venant, en situation d'énonciation dialogique principalement orale, la fonction communicative [Martinet.60, Jakobson.63] du Sling semble prendre une importance prépondérante, en revanche, dans les discours qui sont l'objets de notre recherche, les fonctions heuristiques et démonstratives du Ssém, en situation d'énonciation principalement écrite, «dia-», voire «mono-logique» ${ }^{2}$ deviennent des composantes hautement pertinentes.

5 Ci-dessous, nous proposons deux hypothèses liées au concept de signe, la première résultant de l'interaction entre le Sling et le Ssymb, la seconde de l'utilisation du Ssém comme instrument de recherche et de démonstration, avec des effets illocutionnaires concomitants.

\section{Expansion syntaxique}

6 Avant de présenter nos propres hypothèses, rappelons succinctement l'expansion syntaxique du concept de signe saussurien, liée d'une certaine façon à ces hypothèses, introduite par Igor A. Mel'cuk [Mel'cuk.84] dans le cadre du modèle Sens-Texte.

7 Selon ce modèle, un lexème-signe - correspondant grosso modo à une unité lexicale donnant lieu à une entrée dictionnairique autonome - outre l'image mentale de la forme perceptible, ou signifiant Sa, et le «sens lexical» identifié au signifié Sé, doit comporter des informations - le syntaxique $\mathbf{S t}$ - sur son comportement potentiel dans un contexte limité, d'où la triade formelle ordonnée :

$\mathrm{S}=<\mathrm{Sa}$, Sé, St $>$

Exemples triviaux : l'accord d'un adjectif à un substantif requiert la connaissance du genre grammatical de ce dernier et s'inscrit donc dans le St.

La mise en place des compléments d'un verbe requiert celle de sa rection.

8 En développant la théorie actantielle de Tesnière [Tesnière.53\&59], le modèle Sens-Texte associe à chaque lexème un assortiment de participants sémantiques nécessaires et suffisants pour le caractériser (contenu Sé et fonctionnement $\mathbf{S t}$ ), en l'opposant, notamment, à ses parasynonymes.

Ainsi, le lexème aide, dans: L'aide financière de la Région à l'Association sportive pour la construction de gradins provisoires, fait appel à quatre participants sémantiques :

X, l'agent : «qui aide ?» La Région

11 Y, le bénéficiaire : «à qui est destinée l'aide ?» L'Association sportive

$12 \mathrm{Z}$, l'objet de l'aide : la construction de gradins provisoires

13 W, la manière dont s'effectue cette aide : financière

14 Le syntactique St doit indiquer, entre autres, le régime du substantif aide, c'est-à-dire les moyens morphosyntaxiques nécessaires et suffisants (possibilités et restrictions) permettant d'actualiser dans un discours les participants sémantiques sous forme 
d'actants pris en un sens apparenté à celui de Tesnière. Le segment de texte ci-dessus peut être formalisé comme suit : Aide $\mathrm{W}$ de $\mathrm{X}$ à Y pour $\mathrm{Z}$.

Exemple de restriction. La construction : L'aide de la Région $\underline{a}$ l'Association ... à la construction ... n'est pas acceptable (à cause du redoublement de à), bien que L'aide ... de la Région à l'Association sportive (sans redoublement) le soit.

Les symboles sont également assujettis, dans le cadre d'une discipline donnée, à certaines règles d'assemblage (de concaténation), dites de bonne formation. Peut-on les doter d'un St?

Ainsi, en arithmétique, l'expression "3+)-/14(=" est irrecevable : elle n'est ni vraie ni fausse, elle est dénuée de sens. En revanche l'expression bien formée " $5+5=14$ ", a un sens : elle est fausse dans le système de numération de base10, mais vraie, dans celui de base 6 .

Toujours en arithmétique, le signe «<», de même que le syntagme «est plus petit que» doit être encadré par deux expressions que, métaphoriquement, on peut appeler actants syntaxiques ou participants au niveau sémantique, et lui appliquer le concept de fonction lexicale converse : $\operatorname{Conv}_{21}(<)=>$. Dans le même ordre d'idées, un crochet ouvrant appelle un couple de parenthèses et un crochet fermant : [ $3 \mathrm{x}$ (2 $+5)$ ], mais tel n'est pas obligatoirement le cas en topologie où les crochets ont une toute autre signification : intervalle ouvert ]3,5[, fermé [3,5]

Quels sont les impacts sur la triade $\mathbf{S}$ de l'hétérogénéité du Ssém fonctionnant en situation d'énonciation principalement écrite, avec, comme vocation complémentaire essentielle, la recherche et la démonstration? Par exemple, peut-on concevoir un «Sé de symbole», ne serait-ce que par abus métonymique de langage ${ }^{3}$ ? Peut-on associer un assortiment de participants sémantiques et un syntactique à un symbole donné? L'assortiment est-il identique pour un lexème-mot, comme : courbure, continuité, acide, ébullition, température... et pour le même mot en fonction de terme d'une terminologie? Quelles en sont les conséquences pour les signifiés? Quels sont les non-dits (participants occultables) acceptables en discours? Peut-on parler de force illocutionnaire, voire perlocutionnaire d'un signe-symbole, etc. ?

Pour fixer les idées, donnons quelques exemple simples. Soit l'énoncé attestable ${ }^{4}$ : Point d'ébullition de $\mathrm{H}_{2} 0$ à $\mathrm{T}=373,15 \mathrm{~K}$, sous $\mathrm{p}=1 \mathrm{~atm}$.

$\mathrm{Si}$, communément, ébullition appelle les participants liquide, vapeur, température et, éventuellement, récipient, source de chaleur [CREDIF.76, DESCAMPS.84] ${ }^{5}$, pour le physicien l'absence de pression ôte toute valeur informative à l'énoncé, sauf à accepter comme prérequis (non-dit) l'égalité «p = 1atm». Ceci étant, pour ce même physicien, cette égalité est-elle synonyme de normal dans point normal d'ébullition, car apportant la même information et, par suite, lui étant subtituable dans un tel contexte?

Quel est le contenu illocutionnaire de l'énoncé? Cherche-t-on à informer sur l'existence d'une loi physique, fondamentalement approximative, ou impose-t-on une définition absolue? Comment l'énoncé sera-t-il perçu puis engrangé par l'interlocuteur selon qu'il est spécialiste, apprenant, amateur ou béotien?

La formule $\mathrm{H}_{2} \mathrm{O}$ doit-elle être interprétée dans des contextes spécifiques comme synonyme, à un certain degré d'approximation près, du lexème eau, ou comme abstraction idéelle "antonyme», l'eau ordinaire contenant, entre autres, des impuretés susceptibles d'avoir un effet perturbant (catalyse positive ou négative), selon l'opposition corps pur $\neq$ impur ) ?

La problématique étant trop vaste, nous nous contentons de présenter quelques sujets de discussion.

18 Notre première hypothèse comprend deux faces formant boucle cybernétique (action, rétroaction). D’une part, le Sling exerce une «pression» sur le Ssymb, phénomène bien 
connu en linguistique des textes technoscientifiques [Gentilhomme.68, Kocourek.82]. D'autre part, inversement, le Ssymb a des impacts sur le fonctionnement du Sling, surtout sur le (sous)-système terminologique, la syntaxe et la morphologie paraissant moins concernées.

Bien que l'influence du Ssymb sur la morphologie du sling semble moins importante, citons pour mémoire la formation de lexèmes hétérogènes du Ssém, comme : s-anneau (math.), a-irradié (physique) ... de plus en plus fréquents dans les discours des scientifiques, et, surtout, la terminologie chimique, où l'on trouve de nombreux doublets synonymiques, d'une part, un terme théorique «monstre» (image verbale construite selon des conventions précises d'une formule chimique développée) et, d'autre part, une appellation courante plus synthétique, linguistiquement plus maniable, héritée souvent d'anciennes nomenclatures [DAGOGNET 69]:

méthyl-3 dihydroxy $-1,8$ antraquinone = acide chrysophanique

b-D-fructofurannosyl a-D-glucopyrannoside = saccharose

Etant donné la productivité galopante de telles formations et leur nombre dépassant celui des lexèmes du Sling, il paraît impensable de les traiter comme un phénomène marginal.

Pour préciser les effets de cette interaction, essayons de dégager quelques propriétés spécifiques du Ssymb. Rappelons tout d'abord que, tributaire d'une discipline particulière, chaque Ssém = Sling $\cup$ Ssymb est censé se plier à cette discipline tout en assurant la fonction communicative générale. Les exigences des diverses disciplines variant considérablement, nous avons sciemment circonscrit notre champ d'investigation. Nombre de symboles, ayant atteint une notoriété internationale, même dans des langues écrites en caractères non latins, voire idéographiques, sont originairement des abréviations. Tel est le cas, notammant, de quelques fonctions et concepts mathématiques classiques [Le Lionnais.79]: probabilité, une proposition; de «v» pour un volume, une vitesse ; de «f» pour une force, une fonction, «m» pour une masse, une moyenne, un milieu, etc. L'effet d'hystérésis ${ }^{6}$ joue en faveur du maintien de ces notations par d'autres langues où ces lettres cessent d'être les initiales du terme consacré.

Par une sorte de «métonymie typographique», la motivation se reporte d'abord sur la

variante graphique : lettre majuscule «P», italique «p,» grasse «p», ronde ; sur les mêmes lettres diacritées de diverses façons : p', p", $\underline{p}, p, p$... ou sur les lettres 
correspondantes d'autres alphabets : $\Pi, \Pi, \phi, \Phi, \mu \ldots$ enfin sur les lettres voisines dans l'alphabet considéré : probabilité $\mathrm{q}, \mathrm{r}$, fonction $\mathrm{g}, \mathrm{h}$, etc. $\Sigma$ et $\Pi$ dûment indicées désignent des sommes et des produits d'un grand nombre de termes, voire un nombre infini ; $\int$ sorte de $\langle f »$ déformé - une intégrale, i.e. une certaine somme; $\delta, d-$ une différentielle [Le Lionnais. 79].

Soulignons cependant le fait qu'un changement de corps d'un caractère permet de désigner des référents distincts, parfois apparentés, ce qui, en général ${ }^{7}$, n'est pas le cas dans le sling écrit. La règle de l'initiale majuscule ne joue pas. Une variation du corps, par exemple l'italique, permet, le cas échéant, de distinguer ce qui relève de chacun des systèmes Sling et Ssymb, par ex.: "cri», mot de la langue, de cri, produit des trois facteurs $c, r$ et $i$ ou encore triangle de sommets $c, r$ et $i$. Il existe, par ailleurs, des traditions n'admettant pas de justifications évidentes pour les usagers, du moins en synchronie. Les variables et les inconnues sont souvent désignées par $\mathrm{x}, \mathrm{y}, \mathrm{z}$, puis $\mathrm{u}, \mathrm{v}, \mathrm{w}$; les coefficients par les premières lettres de l'alphabet $a, b, c, \ldots$; les points par des majuscules A, B, C...; les angles par les lettres grecques $\alpha, \beta, \gamma, \phi, \mathrm{v}, \varpi \ldots$

On peut citer des symboles devenus, pour ainsi dire, des noms propres [Le Lionnais.83] : $\pi$ $=3,14159 \ldots$... nombre transcendant [p.80], lié historiquement au cercle; e = 2,7182..., initiale de exponentielle, autre nombre transcendant, base des logarithmes népériens, ou nombre de Neper ; $C=0,5772 \ldots$, constante d'Euler, de nature arithmétique inconnue à ce jour; $\mathrm{i}$ racine de -1 , initiale de imaginaire (remplacée par $\mathrm{j}$ par les électriciens, i réservée pour une intensité de courant) $g \approx 9,81 \mathrm{~m} / \mathrm{s} 2$, la gravité de la Terre; $\mathrm{N} \approx 6,0221 \times 1023$, nombre d'Avogadro, etc.

Cependant, ces motivations et ces usages plus ou moins ancrés n'exercent qu'une incidence pragmatique. En règle générale, le choix de la lettre n'est soumis à aucune obligation, l'«arbitrarité logique» est totale, pourvu que soient respectées les astreintes de :

1. transparence : le «référent» du symbole doit être rendu explicite.

30 2. pérennité : une fois le référent fixé, il doit être rigoureusement maintenu tout au long du discours considéré. Toute modification subreptice, tout dérapage métonymique constituent des fautes graves.

Tolérance. Notons, cependant, que la première astreinte est assouplie par la considération de l'intertexte autorisant des non-dits. Citons, pour éclairer notre propos, les formules classiques telles que $: S=4 \pi R 2$ (géométrie), $\sin (a+b)=\operatorname{sina}$.cosb + sinb.cosa (trigonométrie), $\mathrm{F}=\mathrm{m} \Gamma$ (mécanique), $\mathrm{Va}-\mathrm{Vb}=\mathrm{Ri}$ (électrodynamique), $\mathrm{C}_{2} \mathrm{H}$ ${ }_{5} \mathrm{OH}$ (chimie), $\mathrm{pv}=\mathrm{RT}$ (thermodynamique), $\mathrm{T}=2 \Gamma \pi \sqrt{ } \mathrm{e} / \mathrm{g}$ (mouvements périodiques), etc. Dans la mesure où l'on sait dans quelle discipline on travaille, les notations sont évidentes et les rappels superflus.

Le référent, bien qu'appartenant à un certain domaine sans pour autant être connu de façon précise, peut poser problème. Ainsi, une certaine suite infinie peut théoriquement avoir une limite, que l'on peut nommer sans qu'on sache la déterminer, même de façon approximative.

Le référent peut même s'avérer inexistant dans le domaine considéré, on le désigne néanmoins par un symbole. Voir par ex. la démonstration par l'absurde ou encore la résolution d'une équation, dite improprement «impossible» (pas de solution réelle).

Exemples. «... supposons que les droites $\triangle$ et $\triangle^{\prime}$ se coupent en $M$... il s'ensuit ... ce qui est absurde». Le point $M$ est une entité mythique.

L'équation $\mathrm{X}^{6}+7 \mathrm{X}^{4}+3 \mathrm{X}^{2}+5=0$ n'admet aucune racine (dans $\mathbf{R}$ s'entend, $\mathbf{Z}$ n'étant pas pris en considération). Dans ces conditions que représente $\mathrm{X}$ ? 
Certes, on trouve en Sling des dénominations d'êtres mythiques, comme c'est le cas du dahu (dans le jeu chasse au dahu). Cependant leur fonctionnement discursif est difficilement comparable à celui des symboles, ne serait-ce que par leur faible fréquence.

Concluons que dans le Ssymb l'arbitrarité s'avère bien plus «forte» que dans le Sling.

On voit mal, en effet, qu'au cours d'une conversation normale, autrement que par jeu, un des interlocuteurs invente à tout bout de champ des «signifiants» nouveaux à sa convenance, non liguistiquement motivés, ou décide de désigner, par exemple, par le mot casserole ce que tout un chacun appelle rivière, liberté ou parapluie, même avec les astreintes ci-dessus. Le mathématicien, lui, a parfaitement le droit de désigner par «a», « $\pi » . .$, tout ce que bon lui semble, nonobstant les motivations et les usages. Personne ne lui contestera ce droit, même si les notations inhabituelles paraissent déroutantes et nécessitent une certaine accoutumance.

3 Sans doute, le Sling, comporte quelques «mots jokers», comme machin, truc, chose, tipoter ... ou encore des pronoms et des déictiques dont le contenu n'est déterminé que par le contexte ou la situation. Cependant, il n'y a là rien de comparable au privilège du scientifique qui, par un décret arbitraire, a le droit d'imposer le symbole qu'il juge le plus opportun, ne serait-ce que par pure fantaisie et caprice de l'instant. Dans son discours, les «a», «П»... doivent être tenus pour des Sa ad hoc pour véhiculer les Sé qu'il a en vue, Sé utiles pour développer sa pensée, à l'instant de la production du discours et peut-être au delà. Ceci étant, la durée de vie d'un signe symbole nouvellement créé pourra se limiter aux quelques lignes que l'auteur rédige pour lui-même (monologue) ou pour autrui (dialogue), s'étendre à tout un ouvrage, dépasser les frontières de la communauté à laquelle il appartient, et même passer à la postérité.

Soulignons le caractère illocutoire particulier de cette démarche. C'est à l'instant-même et parce que le scientifique dit (ou écrit) en substance que «a» dénomme telle entité, que «a» dénomme effectivement cette entité. En quelque sorte, sans recourir explicitement à l'expression performative consacrée (je) baptise, il opère en fait un baptême, le lexème performatif proprement dit pouvant rester à l'état latent de non-dit.

L'arbitrarité forte du Ssymb apparaît comme un privilège et l'abandon d'un privilège est toujours frustrant. Mais il y a plus. Il semble difficile d'accepter que des personnes qui utilisent couramment le mode d'expression symbolique ne soient pas influencées, même inconsciemment, par ce mode, tout comme un Parisien qui s'installe à Marseille finit, au fil du temps, par s'imprégner de certains traits comportementaux sociolinguistiques locaux, même si, a priori, il se refuse à adopter l'accent méridional.

face de notre première hypothèse est donc que l'arbitrarité forte du Ssymb instaure par accoutumance un certain état d'esprit libéral, capable d'exercer une pression sur le Sling, plus particulièrement sur le sous-système terminologique. Cette remarque corrobore l'observation qu'un Sa de la langue courante peut être choisi de façon relativement libre pour véhiculer un concept technoscientifique distinct du Sé qui lui est normalement associé.

Notations non-littérales. Outre les lettres appartenant à divers alphabets - latins, grecs, gothiques ( $\mathrm{P}$ ensemble des parties d'un ensemble), hébraïque ( $\mathrm{N}$ aleph, cardinal infini), diversement réalisées et assemblées: $\exists$ (il en existe au moins un), $\forall$ (pour tout), $\mathrm{C}_{\mathrm{p}}^{\mathrm{m}}$ (combinaison de $m$ objets $\mathrm{p}$ à $\mathrm{p}), \in\left(\right.$ de $\varepsilon$, élément de), $\cup$ (union), $\mathrm{e}^{\mathrm{x}}$ (à la puissance $\mathrm{x}$ ), $\log ^{\mathrm{a}}$ (logarithme de base a) - sont adoptés par l'usage courant et, notamment, par des 
manuels scolaires, ou imaginés ad libitum, de nombreux «graphismes» simples ou complexes [Lionnais.79 \& APMEP]. Citons en quelques uns : $=, \equiv, \neq, \approx, \#,<,<,>,+,-, \mathrm{x}, \square$ : / , $\cap, \triangle, *, !, \otimes, \oplus, \notin, \supset, \supseteq, \nabla,($ ) $,[],\{\}, / /, \infty$, etc.

Certains jouent des rôles de prédicats, portant sur des arguments comparables d'un certain point de vue (non orthodoxe) à des participants sémantiques. Certes, l'utilisateur des symboles peut assurer qu'ils ne relèvent que de la syntaxe (système formel) et que l'on n'est nullement obligé de les doter d'un quelconque contenu. Il n'en reste pas moins qu'en mettant en œuvre, par exemple, un signe d'inclusion, en son for intérieur, on peut songer, même si on n'en use pas dans une démonstration logique, aux participants sémantiques contenu/contenant - deux abstractions élaborées psychogénétiquement au fil des ans.

Le substrat psychologique devient patent si l'on songe aux signes issus des pictogrammes en voie de devenir des idéogrammes officialisés, comme // (parallèle), $\perp$ (perpendiculaire), $\angle$ (oblique), $\angle$ (angle), $\triangle$ (triangle), $\rightarrow$ (tend vers)..., passant par les stades de sténo, censurée comme un laisser-aller coupable dans un texte de bonne tenue [Lacombe.64], cependant tolérable dans des notes à usage personnel, finissant, au fil des générations, par acquérir le label de symboles mathématiques.

Performativité. Comme nous l'avons signalé ci-dessus, il existe bien des façons d'imposer à son interlocuteur certaines considérations, en particulier des dénominations, moyennant un signe approprié [Loffler.86]. Rien que le fait d'écrire une égalité comme $\mathrm{E}=$ $\left\{x \mid x^{2}-1>0\right\}$ détermine, dans une situation dialogique, la signification du symbole $E$.

Dans le même ordre d'idées, citons un certain usage des parenthèses: ... la cubique d'équation cartésienne $(\mathrm{x}-\mathrm{a}) \mathrm{y}^{2}+\mathrm{x}^{2}(3 \mathrm{a}+\mathrm{x})=0$ (Trisectrice de Mac Laurin) ...

Dans le Ssém interfèrent deux usages des parenthèses, l'un relevant du Ssymb, l'autre du sling écrit, au même titre que celui des autres signes de ponctuation, comme le tiret ou les deux points. Existe-t-il des symboles attitrés à capacité performative ? Tel semble être le cas en logique du symbole "=déf» (égal par définition). Certes, au cours d'un raisonnement, il peut rappeler une définition antérieure, mais il peut aussi introduire une définition originale, il s'ensuit une procédure intrinséque d'enrichissement du Ssymb en cause.

43 Contre-exemples. Toutefois, l'interaction ne semble pas un phénomène absolu. Chaque système conserve une certaine originalité.

44 1. Mutification. Le concept de variable dite muette, n'intervenant que dans le processus du calcul et non dans le résultat définitif, peut surprendre [Le Lionnais.79].

Dans l'exemple ci-dessous, la lettre "i" est muette et " $\Sigma$ " est le symbole mutifiant :

\begin{tabular}{|c|}
\hline $\mathrm{N}$ \\
\hline$\Sigma=$ ai $(\mathrm{a}-\mathrm{an}+1) /(1-\mathrm{a})$ \\
\hline $\mathrm{i}=1$ \\
\hline
\end{tabular}

Y-a-t-il lieu d'envisager ce concept en discours? En effet, un locuteur utilise constamment de nombreux lexèmes qui n'apparaissent pas dans la conclusion de son raisonnement. Il semble donc que le phénomène de la variable muette du Ssymb soit trivial dans le Sling, donc sans intérêt. Il est vraisemblable qu'il en est de même pour d'autres concepts du Ssymb comme ceux de variable libre ou de variable liée. 
2. Homéostasie. Dans le Sling, comme l'a montré Saussure, la valeur d'un constituant se détermine par opposition à celles de toutes les autres.

A des fins pédagogiques, on peut visualiser cette dépendance en figurant les lexèmes du Sling par des ballons de baudruche entassés dans une boîte. La forme de chaque ballon dépend des ballons voisins, le fait de crever un ballon ou d'en ajouter un perturbe tous les autres, l'espace occupé total restant inchangé.

Il est possible d'interpréter métaphoriquement cet effet comme une autodéfense du Sling contre une agression extérieure (le système veillant en quelque sorte à son «intégrité sémantique») et, par conséquent, de le rattacher au phénomène plus général d'homéostasie.

On peut se poser les questions: en est-il de même du Ssymb? La suppression ou l'adjonction de symbole se répercute-t-elle sur les autres et comment, sachant que les astreintes de transparence et de pérennité interdisent les dérapages clandestins, métonymiques et autres?

Le fait d'appeler $\pi$ une probabilité ou une pression perturbe légèrement le Ssymb. Cette lettre ne peut plus désigner concurremment le nombre transcendant 3,14... (on fait souvent appel à une variante graphique ש). L'impact sur les autres symboles ne se fait guère sentir.

Dans la mesure où les symboles paraissent être faiblement interdépendants, il est juste d'émettre des réserves sur la systémicité $\mathbf{d u}$ «Ssymb», lequel se réduirait à un ensemble faiblement structuré [Gentilhomme.85].

En fait, les problèmes de la cohérence du système, de la création de symboles nouveaux, de l'émergence d'une entité originale doivent être posés dans le cadre de la théorie (du modèle) concernée où la néologie, entre autres, peut, pour diverses raisons, être liée à des bouleversements plus ou moins profonds. Il en est de même pour l'élimination.

Jadis, en trigonométrie, on définissait les fonctions circulaires «séc» et "coséc» (inverses de «sin» et de «cos»); hormis dans certaines applications, elles sont devenues obsolètes, car alourdissant sans grand profit l'arsenal fonctionnel. En revanche, on ne saurait éradiquer sans dommage les concepts et leurs symboles de l'intégrale $\int$ ou du laplacien $\triangle$.

50 Ainsi, le Ssymb ne paraît pas se comporter tout à fait comme le Sling et, si homéostasie il y a dans les deux systèmes (contrairement à l'arbitrarité) il nous paraît prématuré de supposer ici une quelconque influence réciproque. Toutefois, cet aspect de la question relève davantage de l'épistémologie et dépasse de loin notre modeste propos.

51 Conclusion première. Au sein du Ssém, non seulement le Sling a une influence notoire sur le Ssymb, mais inversement, nous postulons que le Ssymb exerce une pression sur le Sling. Cette pression se traduit par une modification de l'arbitrarité du signe - de plus grandes libertés et fantaisies accordées au choix du signifiant, avec en revanche, une rigueur et une pérennité du signifié mieux maîtrisées.

52 En outre, il n'est pas exclu que le Ssymb (cf.: «expressions bien formées») stimule la concision de formulation et la réduction de la syntaxe, mais ces extrapolations débordent notre sujet.

53 Selon une conception largement répandue, la fonction essentielle d'un discours technoscientifique est d'informer. Cette conception, qui n'est pas fausse, a cependant pour effet pervers de détourner l'attention de deux autres fonctions d'une importance capitale : permettre la recherche et la démonstration. 
Sans doute existe-t-il d'autre aspects psycho-sociaux comme: convaincre, intéresser un public plus large de spécialistes, d'amateurs ou de bailleurs de fonds, faire connaître l'auteur, voire discréditer d'autres recherches, etc. Nous ne les rappelons que pour mémoire.

Si l'un des objectifs de la lexicologie peut être formulé, grosso modo, comme suit : étant donné un mot attesté, quel contenu véhicule-t-il ? Comment le définir ? Y a-t-il lieu de distinguer plusieurs acceptions (polysémie, homonymie) ? comment organiser l'article de dictionnaire afférent ? L'objectif du chercheur est tout autre : étant donné une entité qu'il conçoit, comment la désigner? Comment la définir de façon opérationnelle? Inversement, étant donné un terme attesté, quelle est sa définition sine qua non? Où trouver cette définition ? Comment la faire fonctionner au cours d'un raisonnement?

On sait que, pour dénommer une entité, le choix du Sa est en principe logiquement arbitraire, mais humainement motivé. La néologie procède de plusieurs façons et a fait l'objet de multiples travaux. Limitons-nous à citer quelques exemples de termes :

dégénéré, travail, courbure, application, médiatrice, isométrie, ordinateur, taxon, surjection, entropie, hypertextualisation, axiome de Pasch, spirale de Cornu, trou noir, la p'tite dernière ...

et discutons sur un exemple. Considérons l'adjectif dégénéré. Le Petit Robert en présente le sens usuel : qui a perdu les qualités de sa race. Le Larousse propose un contenu plus souple, en substance : abâtardissement, perte de qualités, dégradation. On peut interpréter cette «souplesse» en termes de polysémie n'interdisant pas de regrouper les diverses acceptions voisines dans une même définition. Nous appelons signifié notionnel, Snot, notion en abrégé, l'extension souple, explicitable par des définitions lexicographiques usuelles, complétées en général de citations appropriées.

Si l'on observe ce même adjectif fonctionnant comme terme technoscientifique, force est de constater que son contenu varie de façon discrète (par bonds) d'une discipline à l'autre (mathématiques, physique, biologie, médecine) et ne peut être recouvert par une définition globalisante, sans perdre tout intérêt, même si on retrouve toujours, du moins originairement, l'idée commune de perte d'une qualité importante sans être essentielle. Il s'agit, par exemple, d'une certaine matière formée d'atomes sans électrons périphériques, mais matière quand même. En géométrie, c'est une courbe décomposable en courbes plus élémentaires (une hyperbole dégénère en un couple de droites). En algèbre, on trouve des définitions hermétiques aux non-initiés [Le Lionnais.79], imposant des conditions «mutilantes» aux formes bilinéaires, quadratiques ou sesquilinéaires «normales».

Ces définitions, au sens logique, ne sont que des abréviations de certains assemblages figés du Ssém. Dans chaque cas, l'extension et, conséquemment, la compréhension sont strictement délimitées, la moindre variation engendrant un terme distinct. Nous appelons Signifié conceptuel, Sconc, concept en abrégé, un tel contenu. Tout dérapage sémantique non déclaré constitue une erreur, un canular, voire une malhonnêteté [Gentilhomme.82].

Un romancier peut écrire : «Les associations d'idées se faisaient à la vitesse de la lumière». Une telle assertion déconsidérerait un scientifique au regard de ses pairs. La vitesse de la lumière dans le vide est évaluée actuellement à 299792458m/s. Affirmer que tel est le cas pour signifier que la vitesse paraît grande au regard des vitesses usuelles est inacceptable dans un discours technoscientifique.

Notons que la connaissance d'un Snot, contexte aidant, permet d'appréhender ou, du moins, de subodorer le sens des variantes polysémiques. Tel n'est pas le cas des Sconc de même Sa. Dans l'exemple précédent, l'absence des électrons périphériques ne laisse guère 
deviner la décomposition d'une courbe d'un certain degré en courbes de degré moindre, et, encore moins, qu'une forme bilinéaire possède un noyau réduit à $\{0\}$. Il convient d'opposer polysémie conceptuelle (quasi homonymie) à polysémie notionnelle.

Les définitions rigides sont nécessaires pour assurer la rigueur de la démonstration. On ne peut construire un raisonnement déductif sur une base mouvante, mal assurée. Cependant, elles deviennent castratrices pour l'imagination créative, moteur de la recherche.

Seconde hypothèse. Notre seconde hypothèse est que le chercheur, voire le pédagogue, a concurremment, dans son esprit et le Sconc et le Snot. Le premier, qu'il présente officiellement à ses pairs, est nécessaire pour raisonner correctement; le second, qu'il garde souvent par devers soi en laissant à l'auditoire le soin de le deviner, est utile pour comprendre sa pensée, ce qui en pédagogie peut conduire à des catastrophes. Mais il y aurait trop à en dire et nous ne le pouvons dans le présent article.

61 Ces considérations et bien d'autres nous conduisent à faire éclater le Sé d'un terme technoscientifique :

$S e ́=\langle$ Snot, Sconc $\rangle$, d'où : $S=\langle S a ;$ Snot, Sconc; St $\rangle$

62 Il est clair qu'une justification sérieuse de cette hypothèse exigerait un long développement et que, subséquemment, se posent de multiples questions annexes, comme celles de l'existence d'un Sconc sans Snot, de termes larvés, d'une interaction motivante entre le Sconc et le Sa, de différences dans leur mode d'acquisition, que nous précisons par ailleurs [Gentilhomme. 82,92].10

\section{NOTES}

1. Les Pic de la Mirandole étant inconcevables à notre ère, force est de se rabattre sur le modèle de la termitière proposé par Prigogine et Stenger. Chaque contributeur, selon ses capacités, apporte une boulette parfumée de savoir à l'édifice commun. Nous proposons la nôtre. A la longue, il en resulte une théorie, provisoire, approximative et réductrice comme toutes les théories, exprimant ce qu'il est raisonnable de penser, compte tenu des connaisssances acquises et proposant des moyens heuristiques nouveaux.

2. En matière de recherche, le monologue intérieur secret, relevant pour une part de la psychanalyse, sans qu'on sache vraiment dans quel «langage» il se développe, alterne avec le dialogue ouvert adressé aux pairs et émules, entre autres.

3. Il y a abus de langage de parler de Sé d'un symbole, au sens où l'entendent les sciences formalisées, Sa et Sé n'étant pas indissolublement liés, mais seulement pragmatiquement associés. Néanmoins, en vertu du «principe de continuité», couramment exploité en science, il est commode d'usurper cette terminologie après en avoir avisé le lecteur.

4. Attestable s'oppose à attesté. Dans les sciences exactes, il n'est pas exclu de faire des expériences imaginaires. Les Archimède, Galilée, Einstein ... ne s'en sont pas privés. Le fait d'imaginer un exemple, une situation, même peu probables, peut parfois aider à bien situer un problème. 
5. Les participants sémantiques peuvent être parfois dissimulés dans un vaste contexte et il importe de les débusquer. C'est ce que tentent de faire méthodiquement Descamps et coll. dans l'œuvre remarquable citée ci-dessus.

6. Métaphore utilisée dans le processus d'aimantation et de désaimantation des aimants.

7. Il faut citer cependant l'usage autonyme d'un mot, qui en modifie totalement le contenu.

8. Citons l'expression absurde : «démontrer par $a+b$ » où l'on se fonde naïvement sur l'autorité des mathématiques. Les non scientifiques ont parfois de la peine à accepter qu'un mot courant soit doté d'une signification technique spécifique. Nous nous sommes heurtés à ce fétichisme à propos des mots concept et notion.

9. De semblables bizarreries se rencontrent néanmoins chez certains auteurs. Ainsi André Martel rédige les lettres à ses amis en «paralloïdre»: «Ce primier jodi Florimois aux Vinchênes en Panamie àla Pacificavia; en l'An O du Mirivis. Potami A Tom, Des zonzidisent queuté un mochant. Cèpapossib ...».

10. Au niveau logique, Sconc et Snot s'apparentent respectivement à ensemble structuré (cf. classe distributive) et à classe objet, telle que l'envisage l'Ecole de Neuchâtel (cf. Denis Mieville, «S. Lesniewski ou une manière d'aborder l'ontologie», in Sémiotiques, 1992, n², pp. 28-31). 